\title{
Sleep disorders in pregnancy
}

\author{
Ghada Bourjeily MD
}

Warren Alpert Medical School of Brown University, Pulmonary and Critical Care Medicine, Women \& Infants Hospital, Providence, RI 02905, USA

\begin{abstract}
Summary: Sleep complaints are a common occurrence in pregnancy that are in part due to pregnancy-associated anatomic and physiological changes but may also be due to pathological causes. In the non-pregnant population, sleep deprivation has been associated with physical and cognitive issues; poor sleep may even be associated with adverse maternal outcomes. Maternal obesity, one of the most prevalent risk factors in obstetric practices, together with physiologic changes of pregnancy predispose to the development of sleep disordered breathing. Symptoms of sleep disordered breathing have also been associated with poor maternal outcomes. Management options of restless legs syndrome and narcolepsy pose a challenge in pregnancy; benefits of therapy need to be weighed against the potential harm to the fetus. This article briefly reviews the normal changes in pregnancy affecting sleep, gives an overview of certain sleep disorders occurring in pregnancy, and suggests management options specific for this population.
\end{abstract}

Keywords: sleep medicine, sleep disordered breathing, sleep disorders, obstructive sleep apnoea

\section{BACKGROUND}

Sleep is currently thought to be as complex a state as wakefulness. Animal studies have shown that sleep is also clearly necessary for survival, since prolonged sleep deprivation leads to severe physical and cognitive impairment and, finally, death. Deprivation of a specific component of the sleep cycle known as rapid eye movement (REM) sleep for a few weeks has also been shown to be fatal in rats. ${ }^{1}$ In humans, the consequences of sleep deprivation include daytime sleepiness, fatigue, poor concentration, cognitive dysfunction, poor work performance, impaired immunity, perturbed neuro-endocrine function, insulin resistance, glucose intolerance and possibly obesity.

Sleep is disturbed in pregnancy and the postpartum period for many obvious reasons. Recently, the American Academy of Sleep Medicine has established 'pregnancy-associated sleep disorder' as a separate entity. ${ }^{2}$ This disorder includes both insomnia and excessive sleepiness that develop in the course of pregnancy. There are limited studies looking at the consequences of sleep deprivation in the pregnant population. Recent reports suggest longer duration of labour and a higher rate of caesarean section in a select population of primiparous women with sleep deprivation close to term. ${ }^{3}$ Furthermore, sleep duration of five hours or less six months after delivery has been associated with weight retention of at least $5 \mathrm{~kg}$ at one year postpartum. ${ }^{4}$ Further studies need to be done to assess the effect of sleep deprivation that occurs in pregnancy on cognitive function, mental and physical wellbeing as well as delivery outcomes.

Correspondence to: Ghada Bourjeily

Email: Gbourjeily@wihri.org

\section{NORMAL PREGNANCY-ASSOCIATED CHANGES}

Physiological factors that predispose a pregnant woman to sleep disturbances may be anatomical (discussed in the sleep disordered breathing section), mechanical or hormonal and may develop at different stages of pregnancy.

\section{Mechanical factors affecting sleep initiation and sleep maintenance}

Many changes occur in pregnancy that may lead to difficulties with sleep initiation or sleep maintenance. Gastroesophageal reflux starts early in pregnancy and is related to the relaxation of the lower oesophageal sphincter, delayed gastric emptying and stomach displacement by the enlarging uterus. Consequently, nocturnal symptoms of gastroesophageal reflux due partly to the recumbent position may lead to sleep disruption.

Urine excretion is generally decreased during sleep. However, nocturia is a common occurrence among pregnant women and is related to the increased overnight urine excretion. ${ }^{5}$ Furthermore, decreased bladder capacity secondary to the effects of the enlarged uterus is another cause for frequent micturition occurring both during the day and at night.

Widening of the symphysis pubis, relaxation of the pelvic ligaments as an effect of relaxin ${ }^{6,7}$ with an outward displacement of the hips, all occur in pregnancy. Leg cramps are also common and tend to occur mostly at night. Discomfort related to the above changes may lead to difficulties with sleep initiation, sleep maintenance or both. In addition, sleep in the left lateral decubitus position, nocturnal uterine contractions related to oxytocin peaking at night ${ }^{8}$ and fetal movement may all disrupt sleep. 


\section{Hormonal factors affecting sleep in pregnancy}

Hormones that follow a circadian rhythm may have various effects on sleep. In addition, hormones such as progesterone, oestrogen, cortisol, prolactin, human chorionic gonadotropin and oxytocin are increased in pregnancy and have been shown to have an effect on sleep. Figure 1 summarizes the trend of pregnancy hormones.

Progesterone has sleep-inducing properties and has been repeatedly shown to affect sleep architecture. In fact, subcutaneous injection of pregnenolone (a precursor of progesterone) in rats was shown to enhance slow wave sleep (SWS) as well as increase the amount of REM sleep. ${ }^{9}$

On the other hand, there is ample evidence to suggest that oestrogen reduces REM sleep. ${ }^{10-12}$ REM is enhanced in rats following ovariectomy $^{13}$ and reduced again following oestrogen replacement. In humans, oestrogen replacement has been shown to increase SWS in postmenopausal women ${ }^{14}$ and improve sleep in women without hot flashes. ${ }^{15}$

Prolactin seems to increase SWS (deep sleep) in humans with prolactinoma. ${ }^{16}$ This hormone is also probably responsible for the increase in SWS seen in breast feeding women $(182 \pm 41$ minutes), compared with controls $(86 \pm 22$ minutes, $P<0.001)$ and bottle feeding subjects $(63 \pm 29$ minutes, $P<0.001) .{ }^{17}$
Oxytocin has been shown to peak at night in pregnancy coinciding with the onset of uterine contractions ${ }^{8}$ which may lead to sleep disruption.

Human chorionic gonadotrophin (HCG) rises early in pregnancy and following a central injection of the hormone, rats exhibited a less active and less exploratory behaviour in an open-field box than the control animals. ${ }^{18}$ There are no human studies evaluating the effect of HCG on sleep. Given the thyroid-stimulating properties of $\mathrm{B}-\mathrm{HCG}$, the finding in animals is hard to extrapolate to humans and the effect of this hormone on human sleep remains to be determined.

Other hormones such as cortisol and growth hormone rise in pregnancy and may alter sleep architecture and affect daytime hypersomnolence.

\section{SLEEP DISORDERED BREATHING IN PREGNANCY}

Sleep disordered breathing (SDB) includes the spectrum of snoring, upper airway resistance syndrome and obstructive sleep apnoea (OSA) and hypopnoea syndrome. SDB is a common disorder in both men and women but remains an under-diagnosed disorder in many adults. It is estimated that

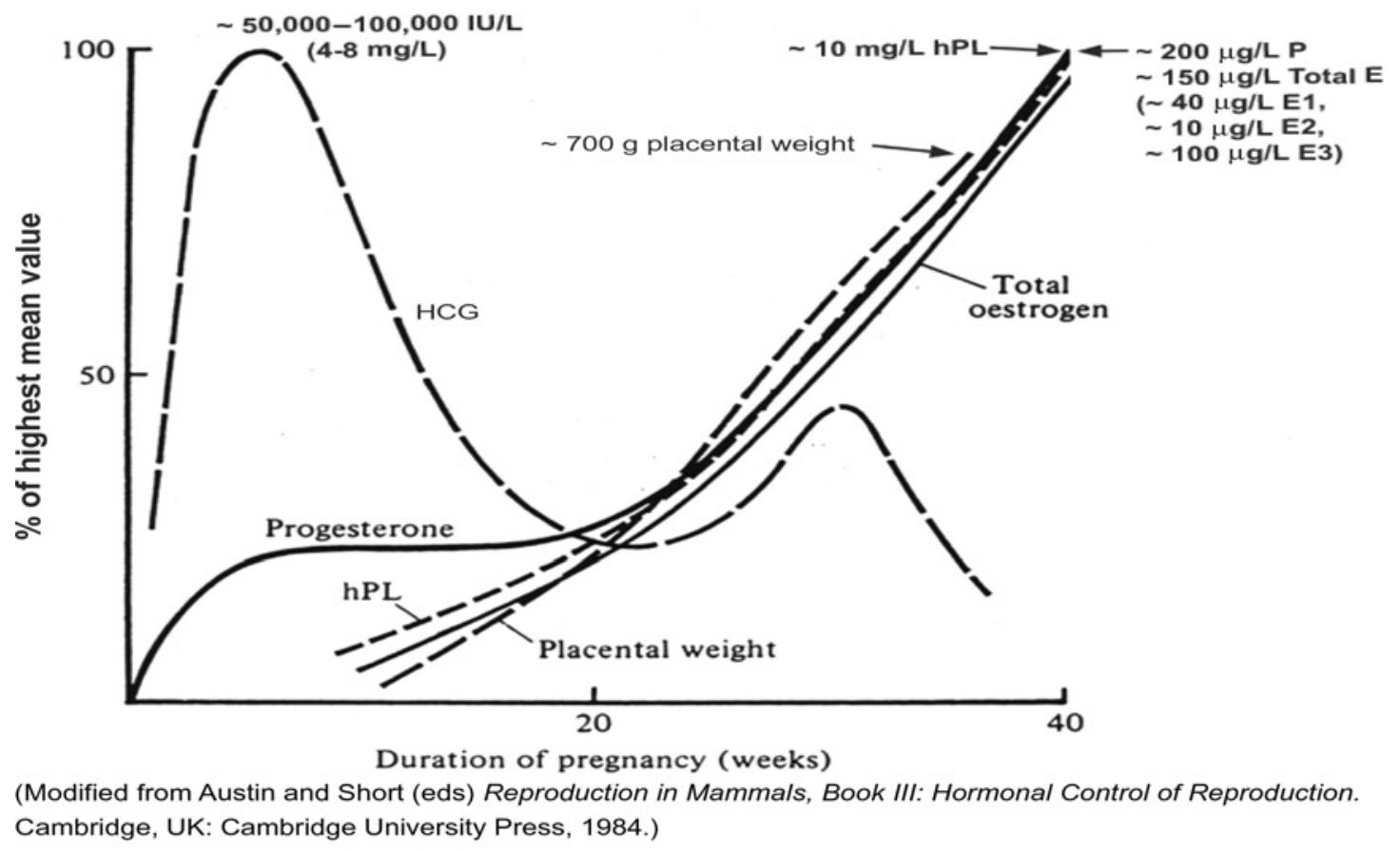

Figure 1 Maternal hormones in human pregnancy. HCG = human chorionic gonadotrophin; hPL = human placental lactogen. Reproduced by permission of Cambridge University Press. 
an apnoea-hypopnoea index (number of apnoeas and hypopnoeas per hour) or AHI over 5 is present in $20 \%$ of the adult population. ${ }^{19}$ However, an $\mathrm{AHI}>5$ combined with one or more of the symptoms of SDB is present in 3-9\% of the nonpregnant population. The prevalence of SDB is two- to threefold higher in patients over 65 compared with patients 30-64 years of age, ${ }^{19}$ and higher in men than women. Although pregnant women fall into the low age risk category, some physiological changes associated with pregnancy may predispose to the development of SDB. It is not clear how physiological changes that increase the risk for the development of SDB are counterbalanced by other factors that may be protective against SDB in pregnancy and what the effect of these changes is on the incidence of SDB in pregnancy.

\section{Effect of pregnancy factors on the development of SDB}

Anatomical changes occurring in the upper airway may predispose to the development of SDB. Hyperaemia and glandular hyperactivity of the upper airway and nasal mucosa as well as increased oedema and friability occur in pregnancy ${ }^{20}$ and are likely to be related to the elevated levels of oestrogens and increased plasma volume. These changes result in what is known as gestational rhinitis, which occurs in over $30 \%$ of pregnant women. Nasal obstruction, especially nocturnal congestion, is an established risk factor for both snoring and SDB in the general population. ${ }^{21,22}$ It is therefore possible that rhinitis of pregnancy could potentially increase the risk of SDB.

Airway size is another risk factor for SDB. Pharyngeal dimensions, assessed using the Mallampati scoring system, are reduced in pregnancy. ${ }^{23}$ More sophisticated measures of upper airway size show a significant reduction at various sites including the oropharyngeal junction in pregnant women compared with nonpregnant controls ${ }^{24}$ and this reduction is even more pronounced in patients with preeclampsia. ${ }^{25}$ Airway narrowing that occurs in normal pregnancy resolved after delivery in this study. ${ }^{24}$

Functional residual capacity (FRC) is reduced in pregnancy and although both minute ventilation and arterial oxygen tension increase in normal gravidas, studies suggest a mild increase in the alveolo-arterial gradient in late pregnancy ${ }^{26}$ and potential desaturations in the supine position related to early airway closing during tidal breathing 27 or to positional changes in cardiac output. In addition, with the reduction in FRC, the caudal traction on the trachea and pharynx is reduced, predisposing to airway collapsibility ${ }^{28}$ but this effect has not been studied specifically in pregnancy. Furthermore, the strong ventilatory drive associated with the increased levels of progesterone may result in a suction effect on the oedematous upper airway, possibly leading to further obstruction. $^{29,30}$

On the other hand, progesterone stimulates the ventilatory drive and increases electromyographic activity of the upper airway dilator muscle. ${ }^{31}$ Progesterone's stimulating properties enhance the responsiveness of the upper airway dilator muscles to chemical stimuli during sleep. ${ }^{32,33}$ This effect of progesterone theoretically protects against the development of SDB and less obstructive events have been observed in 'high progesterone periods' of the menstrual cycle. ${ }^{34}$

The effect of oestrogen on apnoeic/hypopnoeic episodes is not clear since oestrogen replacement therapy reduced the $\mathrm{AHI}$ in patients with mild to moderate $\mathrm{SDB} ;{ }^{35}$ however, drug-induced menopause in healthy premenopausal volunteers failed to show evidence of sleep fragmentation or any significant change in the AHI. ${ }^{36}$

Sleep in the lateral position and the reduction in the time spent in REM sleep in late pregnancy are both protective against apnoeic and hypopnoeic events.

\section{Symptoms of SDB in pregnancy}

The prevalence of SDB in pregnancy has not yet been studied but a few studies have reported on the incidence of symptoms of SDB in this population. Many studies suggest that snoring occurs in $14-45 \%$ of pregnant women ${ }^{37-39}$ as opposed to $4 \%$ of premenopausal women. It is possible that the prevalence of snoring in the general population is an underestimate compared with that in the pregnant population since pregnant women are more likely to have a bed partner, and therefore more likely to be aware of their snoring. However, as a general rule, women tend to under-report snoring. Pregnant women with a higher body mass index (BMI) are more likely to snore than women with a lower BMI. ${ }^{40,41}$ Furthermore, symptoms of daytime hypersomnolence increase in pregnancy, ${ }^{39,42}$ with reported Epworth sleepiness scale (ESS) scores $>10$ in close to $25 \%$ of patients. ${ }^{42}$ Although there are many reasons that could potentially lead to sleep disruption in pregnancy with consequent daytime hypersomnolence, ESS scores correlated with both snoring and gasping in one study $(P=0.0008$ and $P=0.001$, respectively $){ }^{42}$

Although small case-controlled studies show a higher prevalence of OSA by polysomnography in obese pregnant patients than non-pregnant controls, no large-scale epidemiological studies exist to assess the prevalence of this disease in this patient population.

\section{Effect of SDB on the mother and the fetus}

\section{Oxygenation, ventilation and haemodynamic response to apnoea in pregnancy}

The immediate effect of apnoeas on oxygen desaturation and carbon dioxide retention in pregnant women has been studied. Available data have shown a significantly more rapid rate of desaturations ${ }^{43,44}(3.6 \pm 0.8$ versus $7.5 \pm 0.9$ minutes in the pregnant and non-pregnant groups, respectively $)^{44}$ and a faster rise in $\mathrm{CO}_{2}$ in pregnant women compared with nonpregnant controls $(6.8 \pm 1.8 \mathrm{mmHg} /$ minute versus $2.8 \pm$ $1.2 \mathrm{mmHg} /$ minute, respectively), following investigator-induced apnoeas. ${ }^{44}$ However, in this study sedation and paralysis eliminated the effect of the central respiratory drive during obstructive apnoeas. Consequently, this experiment is not an optimal model for apnoeas that occur during sleep.

Hypertensive disorders of pregnancy seem to potentiate the haemodynamic response to apnoeas in pregnancy. In fact, fluctuations in blood pressure readings in response to apnoeic episodes are more pronounced in women with preeclampsia and OSA than women with OSA without hypertension. ${ }^{45}$ Hypertensive fluctuations and the severity of OSA seem to improve in untreated postpartum women. ${ }^{46}$

\section{Pregnancy outcomes of SDB}

Although no large epidemiological studies have evaluated the effect of untreated SDB during pregnancy, there have been 
studies that have evaluated the effect of symptoms of SDB on pregnancy outcomes. Snoring has been shown to be associated with higher rates of pregnancy-induced hypertension and preeclampsia. ${ }^{38,40}$ In a cohort of 502 women, Franklin et al. have shown that snoring was an independent risk factor for both hypertension and preeclampsia, even when adjusted for age and weight. Our data have shown similar findings but also a significant association between poor blood pressure control at two weeks postpartum and symptoms of SDB during pregnancy. ${ }^{40}$

The potential association between preeclampsia and SDB may be even stronger than in normal pregnancy and SDB, given the more prominent changes in upper airway size in women with preeclampsia, as well as the association between hypertension and SDB in the non-pregnant population. Connolly et al. ${ }^{47}$ have demonstrated more significant inspiratory flow limitation in preeclampsia compared with normal pregnant women in the first, second and third trimester and compared with matched non-pregnant women. Small series have found flow limitations in pregnant women with risk factors for preeclampsia. ${ }^{48}$ Data from many case reports or small case series suggest adverse effects of SDB on pregnancy and fetal outcomes. Although these reports may educate the reader on the potential for associations, they do not establish associations and should be interpreted with caution.

Endothelial dysfunction may be a common denominator to SDB and preeclampsia. SDB, regardless of severity, has been shown to be associated with endothelial dysfunction in many studies. Endothelial dysfunction with an imbalance of proangiogenic and antiangiogenic factors is thought to be the underlying mechanism of preeclampsia. Limited data showed that patients with preeclampsia have a higher respiratory disturbance index and a lower endothelial function index when compared with controls. ${ }^{49}$ Further studies need to be done to study potential associations between SDB and pregnancy in general and preeclampsia in particular.

\section{Effect of SDB on the fetus}

Intermittent hypoxia may be the most obvious theoretical mechanism for potential adverse effects on the fetus. Human studies looking at this phenomenon are lacking but limited animal studies showed a reduction in birth weight ${ }^{50}$ in rat pups exposed to intermittent hypoxia in utero, which resolved after 15 days of age. Although epidemiological data from chronically hypoxic pregnant women living at high altitudes strongly suggest a negative effect on intrauterine growth, it is not entirely clear whether the effect of chronic, but intermittent, hypoxia is the same. Case reports have suggested acute fetal decelerations during maternal apnoeic episodes, ${ }^{51-53}$ but those were based on clinical observations of apnoeas and not on polysomnographic data. One recent study has looked at simultaneous polysomnography and fetal non-stress testing and suggested late fetal decelerations accompanying the apnoeic episodes. ${ }^{54}$ However, the study failed to show the temporal relationship between apnoeas, desaturations and decelerations and did not adjust for associated medical problems in the three pregnant women with OSA and fetal decelerations (2 with gestational diabetes mellitus and 1 with cardiovascular disease).

Poor fetal outcomes in women with OSA are suggested by case series and case reports that do not adjust for significant maternal co-morbidities. Data collected on snoring pregnant women and fetal growth restriction are conflicting with case series $^{55}$ and large epidemiological studies ${ }^{38}$ suggesting an association, and others on smaller numbers of patients ${ }^{56,57}$ not supporting this association.

\section{Treatment of SDB in pregnancy}

The decision to treat pregnant women with SDB should probably be made the same way as in the non-pregnant population. Minor differences do, however, exist. Whereas weight loss leads to clinically significant improvement in SDB in the general population, pregnant women should not be asked to lose weight until the postpartum period. Avoidance of drug and alcohol use should be emphasized to pregnant women for many reasons, including their negative effect on symptoms of SDB. Concerns regarding fetal wellbeing in women with recurrent episodes of intermittent apnoea and potential intermittent desaturations and hypoventilation should be taken into consideration, despite the lack of studies directly linking the two processes.

A review of prescribed medications that could affect sleep architecture or contribute to sleep symptoms should be a routine part of the evaluation. Most experts agree to treat symptomatic non-pregnant patients with an apnoea-hypopnoea index greater than 5 per hour. Although no recommendations exist for the pregnant population specifically, it is reasonable to follow those same guidelines in pregnancy.

Continuous positive airway pressure (CPAP) is the preferred method of treatment of SDB in the general population and helps treat over $90 \%$ of patients with OSA. Although there is a theoretical concern regarding an increased risk of aspiration in pregnancy with the use of CPAP, in our experience it is well tolerated in pregnancy and does not seem to be associated with a higher risk of complications in this population. CPAP pressures may have to be titrated again during the course of pregnancy because of weight gain but the required increase in pressures may only be of $1-2 \mathrm{~cm}$. Auto-titrating CPAP equipment may be used instead of repeat polysomnography and may be a more convenient method since it may be done at the patient's home.

Both the acute and the chronic effects of CPAP have been studied in subgroups of pregnant patients. In the acute setting, the effect of CPAP on haemodynamic parameters has been studied in patients with severe preeclampsia. ${ }^{58,59} \mathrm{CPAP}$ therapy is associated with lower nocturnal blood pressure measurements in patients with preeclampsia during the treatment night in all stages of sleep $^{58}$ (see Table 1). CPAP therapy also improves nocturnal cardiac output on the treatment night in patients with preeclampsia when compared with a control preeclampsia group that had a repeat polysomnography and no CPAP therapy ${ }^{59}$ (see Figure 2).

In a small study, patients with risk factors for preeclampsia and flow limitation on baseline polysomnogram were started on CPAP therapy at the first prenatal visit and continued therapy until delivery. ${ }^{48}$ None of the patients with chronic hypertension in this study developed preeclampsia or needed blood pressure medication adjustment and all women exhibited the normal dip in nocturnal blood pressure. Another study by the same authors randomized patients with risk factors for preeclampsia to either receive usual care plus nocturnal CPAP or usual care alone starting in early pregnancy. ${ }^{60}$ Patients on CPAP exhibited significantly lower systolic and diastolic 
Table 1 Effect of CPAP therapy on nocturnal blood pressure measurements in patients with preeclampsia ${ }^{58}$

\begin{tabular}{|c|c|c|c|c|c|c|c|c|}
\hline & \multicolumn{2}{|c|}{ Stages $1 / 2$ NREM sleep } & \multicolumn{2}{|c|}{ Stages 3/4 NREM sleep } & \multicolumn{2}{|c|}{ REM sleep } & \multicolumn{2}{|c|}{ All sleep } \\
\hline & SBP & DBP & SBP & DBP & SBP & DBP & SBP & DBP \\
\hline Non-treatment & $137 \pm 4$ & $81 \pm 2$ & $144 \pm 4$ & $88 \pm 2$ & $140 \pm 6$ & $84 \pm 5$ & $139 \pm 4$ & $83 \pm 2$ \\
\hline Treatment & $128 \pm 3$ & $74 \pm 3$ & $130 \pm 5$ & $76 \pm 3$ & $131 \pm 4$ & $76 \pm 3$ & $129 \pm 4$ & $73 \pm 3$ \\
\hline$P$ value & 0.005 & 0.017 & 0.015 & 0.039 & 0.019 & 0.032 & 0.006 & 0.004 \\
\hline
\end{tabular}

Reproduced by kind permission of the American Thoracic Society

blood pressure measurements throughout pregnancy and none of the seven patients on therapy developed preeclampsia. Interestingly, APGAR scores were significantly higher in the treatment group at one minute compared with the placebo group. ${ }^{60}$

In summary, although the studies mentioned above involve only a small number of subjects, they are supportive of a potential haemodynamic benefit to the use of CPAP therapy in patients with preeclampsia.

Other therapies for OSAs include oral appliances that have a well-documented benefit in eliminating apnoeas in patients with mild to moderate disease. The effect of this therapy on patients with severe disease is less well documented. If a pregnant patient is to be treated with an oral appliance, it is reasonable to repeat polysomnography on treatment to document resolution of both apnoeas and desaturations.

The severity of SDB may improve following delivery and a re-evaluation of symptoms as well as daytime sleepiness should be done in the postpartum period. This evaluation should take into consideration that sleep may be quite fragmented in the postpartum period but improves in most cases after 3-6 months. Repeat polysomnography may be necessary postpartum to evaluate patients in the absence of physiological factors associated with pregnancy that may have predisposed to the development of SDB. Although not all sleep apnoea cases will resolve postpartum, those that do persist in the postpartum period may need a different CPAP prescription following weight loss.

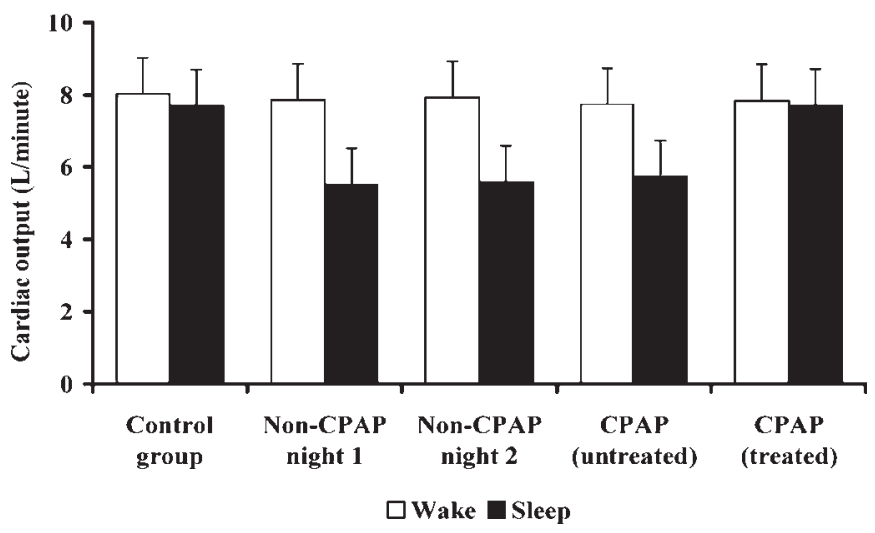

Figure 2 Effect of CPAP on nocturnal cardiac output. CPAP $=$ continuous positive airway pressure.Reproduced with permission. Blyton DM, Sullivan CE, Edwards N. Reduced nocturnal cardiac output associated with preeclampsia is minimized with the use of nocturnal nasal CPAP. Sleep 2004;27(1):79-84

\section{RESTLESS LEGS SYNDROME}

Restless legs syndrome (RLS) is characterized by an unpleasant leg sensation that causes an almost irresistible urge to move the legs. This sensation occurs usually prior to sleep onset. RLS is a sensorimotor disorder where the sensory component is the urge to move the legs and the motor component of RLS is the fact that movement often relieves the urge to move the legs and eliminates the discomfort. A diagnosis of RLS is established when four clinical features are present: $(1)$ the urge to move the legs; (2) the urge is worse when the patient is sedentary; (3) the abnormal sensation in the legs or the urge to move improves or resolves when movement of the legs occurs; and (4) the symptoms have a circadian pattern and get worse during the evening or night.

RLS occurs more frequently in pregnancy than in the general population and is reported in as many as $27 \%$ of pregnant women. ${ }^{61}$ RLS is classified as primary (idiopathic) or secondary. When RLS is associated with pregnancy, this syndrome is considered secondary. Other causes of secondary RLS include iron deficiency anaemia, folate deficiency, advanced renal disease and peripheral neuropathy, and those should be considered in the work-up of pregnant women with RLS. Other less common associations include Parkinson's disease, rheumatoid arthritis and fibromyalgia.

The underlying mechanism for RLS in the non-pregnant population is thought to be mainly related to dopaminergic insufficiency. Iron deficiency probably plays a role as well. The mechanism for RLS in pregnancy possibly involves folate metabolism. This theory is supported by the fact that folate requirements are higher in pregnancy and that replacement therapy may help with resolution of symptoms. Some evidence suggests a lower plasma folate level in patients with RLS compared with those without the syndrome. In a small study of 21 pregnant patients, ${ }^{62}$ patients randomized to receiving folate supplementation in addition to their multivitamin had a much lower incidence of RLS (9\%) when compared to those who did not receive folate supplementation $(80 \%)$. The strikingly elevated prevalence of RLS in the control group challenges the validity of these results.

Other theories in pregnancy include iron deficiency and dopamine insufficiency as well as hormonal causes related to progesterone and prolactin.

Treatment of RLS in pregnancy aims at identifying iron or folate deficiency and replenishing the stores. Although the mainstay of therapy for this syndrome is monotherapy with levodopa and dopaminergic agents, levodopa has been associated with adverse pregnancy outcomes in experimental animals after high-dose treatment. Safety in human pregnancies has not been established despite case reports in humans describing no evidence of abnormal embryological or fetal development. 
There are no data to support the safety of pramipexole or ropirinole use in pregnancy. Benzodiazepine and opiate use can be justified in very symptomatic patients after organogenesis but those should be used with extreme caution close to term as neonatal withdrawal may occur. Clonidine use has been described and may be a potential option in pregnant women as well.

\section{NARCOLEPSY}

Narcolepsy is a disorder of sleep-wake state control where elements of sleep intrude into wakefulness and elements of wakefulness intrude into sleep. Narcolepsy is the second most common cause of disabling daytime hypersomnolence after sleep apnoea ${ }^{63}$ and affects $0.25-0.5$ per 1000 people. Women of childbearing age may be affected as the disease typically manifests itself in the teens to early twenties.

Narcolepsy manifests as excessive daytime sleepiness, cataplexy, sleep paralysis and hypnogogic hallucinations. Excessive daytime sleepiness can be assessed using the ESS, which is a questionnaire that assesses chances of falling asleep during daily activities. Cataplexy, an emotionally triggered loss of muscle tone or weakness, may be partial, affecting specific muscles such as the face, the neck or the knees, or more generalized causing complete collapse. Cataplexy usually occurs within 3-5 years of the onset of hypersomnolence in about $60 \%$ of patients with narcolepsy. ${ }^{64}$ Sleep paralysis is a complete inability to move for one to two minutes after awakening and may be accompanied by hallucinations. Hallucinations may also occur at sleep onset.

Narcolepsy is a result of the loss of the neuropeptides orexin A and orexin B (also known as hypocretin 1 and 2). Orexin inhibits REM sleep and loss of orexin may allow REM-sleep-related phenomena such as cataplexy and hypnagogic hallucinations to intrude into wakefulness. Although genetic factors predispose to the development of narcolepsy, environmental factors may play an even more important role in the pathogenesis, as only $25 \%$ of monozygotic twins are concordant for narcolepsy. ${ }^{65}$

A multiple sleep latency test helps confirm the diagnosis of narcolepsy and a polysomnogram helps exclude coexisting causes of sleepiness that may require specific therapy such as sleep apnoea or periodic limb movement disorder.

Data on narcolepsy in pregnancy are lacking except for some case reports. The diagnosis should be suspected in pregnant patients with daytime hypersomnolence that is out of the ordinary for the 'physiological' sleepiness of pregnancy. Patients with persistent or excessive hypersomnolence should be evaluated the same way as non-pregnant patients.

Patients with narcolepsy should be counselled to avoid prescribed as well as over-the-counter drugs that may induce daytime sleepiness or cause insomnia, and avoid sleep deprivation altogether as this may worsen symptoms of narcolepsy. Scheduled once or twice daily, 20-minute naps often reduce hypersomnolence for up to three hours.

Pharmacotherapy should be considered in patients with persistent symptoms despite the above measures. Modafinil, a non-amphetamine 'wakefulness-promoting agent', has become the first-line therapy in non-pregnant patients with narcolepsy because of its efficacy and tolerability. Data on its use in pregnancy are limited to animal reports of teratogenicity at $200 \mathrm{mg} / \mathrm{kg} /$ day but not at $100 \mathrm{mg} / \mathrm{kg} /$ day. There are also some limited case reports on its safe use in human pregnancy.

Other drugs such as methylphenidate and amphetamines are considered second-line agents, given their sympathomimetic side-effects. Data regarding their safety in pregnancy is also quite limited.

Despite the limited safety profile of these drugs in pregnancy, risk versus benefit should be weighed. Patients with a history of complete collapse related to cataplexy may place themselves as well as their unborn child at risk of potential trauma and should probably be prescribed therapy despite the lack of safety data, with proper counselling. On the other hand, patients with less than severe daytime hypersomnolence and partial cataplexy may benefit from timed naps during the day and may be kept off drugs during pregnancy. Selective serotonin re-uptake inhibitors (SSRIs) have a REM suppressing effect and are beneficial in the treatment of cataplexy. Use of these drugs in the second half of pregnancy may be associated with transient neonatal complications such as mild respiratory distress and jitteriness but also with more severe complications that include higher rates of admission to the neonatal intensive care unit and persistent neonatal pulmonary hypertension. ${ }^{66}$ The use of SSRIs may be justified in patients with depression since depression in pregnancy is associated with poor pregnancy and fetal outcomes and SSRIs reduce the risk of relapse. The risk of untreated cataplexy in an individual pregnant patient needs to be weighed against the risk associated with the drug itself.

\section{CONCLUSION}

Sleep complaints are a common occurrence in pregnancy and are not always related to normal physiological changes. Given that (1) symptoms of SDB are common in pregnancy and seem to be associated with adverse pregnancy outcomes, (2) narcolepsy is a disorder of the young population and (3) restless legs are a frequent complaint in pregnant women, a systematic assessment of the sleep history should be performed in pregnant women to evaluate for these disorders.

\section{REFERENCES}

1 Rechtschaffen A, Bergmann BM, Everson CA, et al. Sleep deprivation in the rat: X. Integration and discussion of the findings. Sleep 1989;12:68

2 American Academy of Sleep Medicine. International Classification of Sleep Disorders, revised: Diagnostic and Coding Manual. Westchester, IL: American Academy of Sleep Medicine, 2000:114-5

3 Lee KA, Gay CL. Sleep in late pregnancy predicts length of labor and type of delivery. Am J Obstet Gynecol 2004;191:2041-6

4 Gunderson EP, Rifas-Shiman SL, Oken E, et al. Association of fewer hours of sleep at 6 months postpartum with substantial weight retention at 1 year postpartum. Am J Epidemiol 2008;167:178-87

5 Parboosingh J, Doig A. Studies of nocturia in normal pregnancy. BJOG: Int J Obstet Gynaecol 1973;80:888-95

6 Goldsmith LT, Weiss G, Steinetz BG. Relaxin and its role in pregnancy. Endocrinol Metab Clin North Am 1995;24:171-86

7 Sherwood OD, Downing SJ, Guico-Lamm ML, Hwang JJ, O’Day-Bowman MB, Fields PA. The physiological effects of relaxin during pregnancy: studies in rats and pigs. Oxf Rev Reprod Biol 1993;15:143-89

8 Hirst JJ, Haluska GJ, Cook MJ, Hess DL, Novy MJ. Comparison of plasma oxytocin and catecholamine secretions with uterine activity in pregnant rhesus monkeys. J Clin Endocrinol Metab 1991;73:804-10

9 Lancel M, Cronlein TA, Muller-Preuss P, Holsboer F. Pregnenolone enhances EEG delta activity during non-rapid eye movement sleep in the rat, in contrast to Midazolam. Brain Res 1994;646:85-94

10 Colvin GB, Whitmoyer DI, Lisk RD, Walter DO, Sawyer CH. Changes in sleep-wakefulness in female rats during circadian and estrus cycles. Brain Res 1968;7:173-83

11 Branchey M, Branchey L, Nadler RD. Effects of estrogen and progesterone on sleep patterns of female rats. Physiol Behav 1971;6:743-6

12 Fang J, Fishbein W. Sex differences in paradoxical sleep: influence of estrus cycle and ovariectomy. Brain Res 1996;734:275-85 
13 Colvin GB, Whitmoyer DI, Sawyer CH. Circadian sleep-wakefulness patterns in rats after ovariectomy and treatment with estrogen. Exp Neurol 1969;25:616-25

14 Leproult R, Hoffmann E, Plat L, van Cauter E. Slow wave activity: effects of gender, age, and estrogen replacement therapy. Sleep 1998;21:300

15 Polo-Kantola P, Efkkola R, Helenius H, Irjala K, Polo O. When does estrogen replacement therapy improve sleep quality? Am J Obstet Gynecol 1998;178:1002-9

16 Frieboes RM, Murck H, Stalla GK, Antonijevic IA, Steiger A. Enhanced slow wave sleep in patients with prolactinoma. J Clin Endocrinol Metab 1998;83: 2706-10

17 Blyton DM, Sullivan CE, Edwards N. Lactation is associated with an increase in slow-wave sleep in women. J Sleep Res 2002;11:297-303

18 Lukacs H. Rat as model for studying behavior effects of hCG. Sem Reprod Med 2001:19:111-9

19 Punjabi NM. The epidemiology of adult obstructive sleep apnea. Proc Am Thorac Soc 2008;5:136-43

20 Camann WR, Ostheimer GW. Physiological adaptation during pregnancy. Intern Anesthes Clin 1990;28:2-10

21 Young T, Finn L, Palta M. Chronic nasal congestion at night is a risk factor for snoring in a population-based cohort study. Arch Intern Med 2001;161:1514-9

22 Young T, Finn L, Kim H. Nasal obstruction is a risk factor for sleep disordered breathing. The University of Wisconsin Sleep and Respiratory Research Group. J Allergy Clin Immunol 1997;99:S757-62

23 Pilkington S, Carli F, Dakin MJ, et al. Increase in Mallampati score during pregnancy. Br J Anesth 1995;74:638-42

24 Izci B, Vennelle M, Liston WA, Dundas KC, Calder AA, Douglas NJ. Sleep disordered breathing and upper airway size in pregnancy and post-partum. Eur Respir J 2006;27:321-7

25 Izci B, Riha RL, Martin SE, et al. The upper airway in pregnancy and pre-eclampsia. Am J Respir Crit Care Med 2003;167:137-40

26 Crapo RO. Normal cardiopulmonary physiology during pregnancy. Clin Obstet Gynecol 1996;39:3-16

27 Garrard GS, Littler WA, Redman CW. Closing volume during normal pregnancy. Thorax 1978;33:488-92

28 White DP. Pathogenesis of obstructive and central sleep apnea. Am J Respir Crit Care Med 2005;172:1363-70

29 Santiago JR, Nolledo MS, Kinzler W, Santiago TV. Sleep and sleep disorders in pregnancy. Ann Intern Med 2001;134:396-408

30 Edwards N, Middleton PG, Blyton DM, Sullivan CE. Sleep disordered breathing and pregnancy. Thorax 2002;57:555-8

31 Popovic RM, White DP. Upper airway muscle activity in normal women: influence of hormonal status. J Appl Physiol 1998;84:1055-62

32 Parisi RA, Santiago TV, Edelman NH. Genioglossal and diaphragmatic EMG responses to hypoxia during sleep. Am Rev Respir Dis 1988;138:610-6

33 Wheatley JR, White DP. The influence of sleep on pharyngeal reflexes. Sleep 1993;16:587-9

34 Stahl ML, Orr WC, Males JL. Progesterone levels and sleep-related breathing during menstrual cycles of normal women. Sleep 1985;8:227-30

35 Manber R, Kuo TF, Cataldo N, Colrain IM. The effects of hormone replacement therapy on sleep-disordered breathing in postmenopausal women: a pilot study. Sleep 2003;26:163-8

36 D'Ambrosio C, Stachenfeld NS, Pisani M, Mohsenin V. Sleep, breathing and menopause: the effect of fluctuating estrogen and progesterone on sleep and breathing in women. Gend Med 2005;2:238-45

37 Schutte SDA, Gross A, Segal J, et al. Self reported snoring and sleep in high risk pregnancies. Sleep Res 1995;24:342

38 Franklin KA, Holmgren PA, Jonsson F, Poromaa N, Stenlund H, Svanborg E. Snoring, pregnancy-induced hypertension, and growth retardation of the fetus. Chest 2000;117:137-41

39 Pien GW, Fife D, Pack AI, Nkwuo JE, Schwab RJ. Changes in symptoms of sleep-disordered breathing during pregnancy. Sleep 2005;28:1299-305

40 Bourjeily G, Habr F, Miller M, Rosene-Montella K. Symptoms of sleep disordered breathing, BMI and pregnancy outcomes. Accepted for slide presentation at the American College of Chest Physicians for 2007 meeting, Chicago, IL. Chest 2007;132:p463a

41 Maasilta P, Bachour A, Teramo K, Polo O, Laitinen LA. Sleep-related disordered breathing during pregnancy in obese women. Chest 2001;120:1448-54
42 Bourjeily G, Raker C. Epworth Sleepiness Score in pregnancy. American Thoracic Society in Toronto, 2008

43 Archer GW Jr, Marx GF. Arterial oxygen tension during apnoea in parturien women. Br I Anaesth 1974;46:358-60

44 Cheun JK, Choi KT. Arterial oxygen desaturation rate following obstructive apnea in parturients. J Korean Med Sci 1992;7:6-10

45 Edwards N, Blyton DM, Kirjavainen TT, Sullivan CE. Hemodynamic responses to obstructive respiratory events during sleep are augmented in women with preeclampsia. Am J Hypertens 2001;14(11 Pt 1):1090-5

46 Edwards N, Blyton DM, Hennessy A, Sullivan CE. Severity of sleepdisordered breathing improves following parturition. Sleep 2005;28:737-41

47 Connolly G, Razak AR, Hayanga A, Russell A, McKenna P, McNicholas WT. Inspiratory flow limitation during sleep in pre-eclampsia: comparison with normal pregnant and nonpregnant women. Eur Respir J 2001;18:672-6

48 Guilleminault C, Palombini L, Poyares D, Takaoka S, Huynh NT, El-Sayed Y. Pre-eclampsia and nasal CPAP: part 1. Early intervention with nasal CPAP in pregnant women with risk-factors for pre-eclampsia: preliminary findings. Sleep Med 2007;9:9-14

49 Yinon D, Lowenstein L, Suraya S, et al. Pre-eclampsia is associated with sleep-disordered breathing and endothelial dysfunction. Eur Respir J 2006;27: 328-33

50 Gozal D, Reeves SR, Row BW, Neville JJ, Guo SZ, Lipton AJ. Respiratory effects of gestational intermittent hypoxia in the developing rat. Am J Respir Crit Care Med 2003;167:1540-7

51 Joel-Cohen SJ, Schoenfeld A. Fetal response to periodic sleep apnea: a new syndrome in obstetrics. Eur J Obstet Gynecol Reprod Biol 1978;8:77-81

52 Roush SF, Bell L. Obstructive sleep apnea in pregnancy. J Am Board Fam Pract 2004;7:292-4

53 Charbonneau M, Falcone T, Cosio MG, Levy RD. Obstructive sleep apnea during pregnancy: therapy and implications for fetal health. Am Rev Respir Dis 1991;144:461-3

54 Sahin FK, Koken G, Cosar E, et al. Obstructive sleep apnea in pregnancy and fetal outcome. International Journal of Gynaecology $\mathcal{E}$ Obstetrics 2008; 100:141-6

55 Schoenfeld A, Ovadia Y, Neri A, Freedman S. Obstructive sleep apnea (OSA)-implications in maternal-fetal medicine. A hypothesis. Med Hypotheses 1989;30:51

56 Guilleminault C, Querra-Salva M, Chowdhuri S, Poyares D. Normal pregnancy, daytime sleeping, snoring and blood pressure. Sleep Med 2000;1:289-97

57 Hedman C, Pohjasvaara T, Tolonen U, Suhonen-Malm AS, Myllyla VV. Effects of pregnancy on mothers' sleep. Sleep Med 2002;3:37-42

58 Edwards N, Blyton DM, Kirjavainen T, Kesby GJ, Sullivan CE. Nasa continuous positive airway pressure reduces sleep-induced blood pressure increments in preeclampsia. Am I Respir Crit Care Med 2000;162:252-7

59 Blyton DM, Sullivan CE, Edwards N. Reduced nocturnal cardiac output associated with preeclampsia is minimized with the use of nocturnal nasal CPAP. Sleep 2004;27:79-84

60 Poyares D, Guilleminault C, Hachul H, et al. Pre-eclampsia and nasal CPAP part 2. Hypertension during pregnancy, chronic snoring, and early nasal CPAP intervention. Sleep Med 2007;9:15-21

61 Manconi M, Govoni V, De Vito A, et al. Restless legs syndrome and pregnancy. Neurology 2004;63:1065-9

62 Botez MI, Lambert B. Folate deficiency and restless-legs syndrome in pregnancy. N Engl J Med 1977;297:670

63 Zeman A, Britton T, Douglas N, et al. Narcolepsy and excessive daytime hypersomnolence. BMJ 2004;329:724-8

64 Okun ML, Lin L, Pelin Z, et al. Clinical aspects of narcolepsy-cataplexy across ethnic groups. Sleep 2002;25:27-35

65 Mignot E. Genetic and familial aspects of narcolepsy. Neurology 1998;50: S16-22

66 American College of Obstetricians and Gynecologists Committee on Obstetric Practice. Treatment with selective serotonin reuptake inhibitors during pregnancy. ACOG committee opinion No. 354. Obstet Gynecol 2006;108: 1601-3 